Aksaray University
Journal of Science and Engineering
e-ISSN: 2587-1277
http://dergipark.gov.tr/asujse
$\underline{\text { http://asujse.aksaray.edu.tr }}$

Research Article

\title{
A Note on Modified Pell Polynomials
}

Nusret Karaaslan*

Aksaray University, Faculty of Science and Letters, Department of Mathematics, Aksaray, 68100, Turkey

-Received Date: 11 Jan $2019 \quad$-Revised Date:9 Feb $2019 \quad$-Accepted Date:13 Feb $2019 \quad$-Published Online:23 May 2019

\begin{abstract}
In this paper, we study the modified Pell polynomials. We first give the proof of the generating function of these polynomials. We then give the proof of the Binet formula for the modified Pell polynomials, which gives the $n$th modified Pell polynomial. We also obtain some summation formula for these polynomials. In addition, we investigate some well-known identities including Catalan, Cassini, d'Ocagne and Gelin-Cesaro identities involving the modified Pell polynomials.
\end{abstract}

\section{Keywords}

Modified Pell sequence, Modified Pell polynomial sequence

\section{INTRODUCTION}

In recent years, the second-order recurrent sequences have been studied by many authors. The well-known examples of these sequences are Fibonacci, Lucas, Pell, Pell-Lucas and modified Pell. We refer the reader to [1-7].

In Ref. [2], the Fibonacci and Lucas sequences $\left\{F_{n}\right\}$ and $\left\{L_{n}\right\}$ are defined by the recurrence relations

$$
\begin{aligned}
& F_{0}=0, \quad F_{1}=1, \quad F_{n}=F_{n-1}+F_{n-2} \text { for } n \geq 2, \\
& L_{0}=2, \quad L_{1}=1, \quad L_{n}=L_{n-1}+L_{n-2} \text { for } n \geq 2,
\end{aligned}
$$

respectively.

*Corresponding Author: Nusret Karaaslan, nusret5301@gmail.com 
In Ref. [7], the Pell, Pell-Lucas and modified Pell sequences $\left\{P_{n}\right\},\left\{Q_{n}\right\}$ and $\left\{q_{n}\right\}$ are defined by the recurrence relations

$$
\begin{gathered}
P_{0}=0, \quad P_{1}=1, \quad P_{n}=2 P_{n-1}+P_{n-2} \text { for } n \geq 2, \\
Q_{0}=2, \quad Q_{1}=2, \quad Q_{n}=2 Q_{n-1}+Q_{n-2} \text { for } n \geq 2, \\
q_{0}=1, \quad q_{1}=1, \quad q_{n}=2 q_{n-1}+q_{n-2} \text { for } n \geq 2,
\end{gathered}
$$

respectively.

Various sequences of polynomials by the names of Fibonacci and Lucas polynomials occur in the literature over a century. The Fibonacci polynomials $F_{n}(x)$ are defined by the recurrence relation

$$
F_{n}(x)=x F_{n-1}(x)+F_{n-2}(x)
$$

where $F_{0}(x)=0, F_{1}(x)=1$ and $n \geq 2$.

The Lucas polynomials $L_{n}(x)$ are defined by

$$
L_{n}(x)=x L_{n-1}(x)+L_{n-2}(x)
$$

where $L_{0}(x)=2, L_{1}(x)=x$ and $n \geq 2$.

The Fibonacci and Lucas polynomials have many properties which have been studied in [8-11]. In Ref. [12], Horadam and Mahon introduced Pell and Pell-Lucas polynomials. The Pell and Pell-Lucas polynomial sequences are $P_{n}(x)$ and $Q_{n}(x)$ are defined by the recurrence relations

$$
\begin{gathered}
P_{0}(x)=0, \quad P_{1}(x)=1, \quad P_{n}(x)=2 x P_{n-1}(x)+P_{n-2}(x) \text { for } n \geq 2, \\
Q_{0}(x)=2, \quad Q_{1}(x)=2 x, \quad Q_{n}(x)=2 x Q_{n-1}(x)+Q_{n-2}(x) \text { for } n \geq 2,
\end{gathered}
$$

respectively.

Additionally, as a special case of Horadam polynomials [13], the modified Pell polynomials are defined recursively by,

$$
q_{0}(x)=1, \quad q_{1}(x)=x ; \quad q_{n}(x)=2 x q_{n-1}(x)+q_{n-2}(x) .
$$

Also, the Binet formula and generating function of these polynomials gave by the authors in the same paper without proofs, respectively, as

$$
\begin{gathered}
q_{n}(x)=x \frac{\alpha^{n}(x)+\beta^{n}(x)}{\alpha(x)+\beta(x)} \\
f(t, x)=\frac{1-x t}{1-2 x t-t^{2}} .
\end{gathered}
$$

The main objective of this paper is to study modified Pell polynomials. 


\section{ON THE MODIFIED PELL POLYNOMIALS}

In this section, we first give the proof of the generating function and Binet formula of the modified Pell polynomials. Then, we obtain summation formulas and various identities for this sequence.

Firstly, we aim to give the proof of the generating function for the modified Pell polynomials.

Theorem 1. The generating function of the modified Pell polynomials is

$$
f(t, x)=\frac{1-x t}{1-2 x t-t^{2}}
$$

Proof of Theorem 1. The generating function can be written as $f(t, x)=\sum_{n=0}^{\infty} q_{n}(x) t^{n}$.

Then we have,

$$
\begin{gathered}
f(t, x)=q_{0}(x)+q_{1}(x) t+q_{2}(x) t^{2}+\cdots+q_{n}(x) t^{n}+\cdots \\
2 x t f(t, x)=2 x q_{0}(x) t+2 x q_{1}(x) t^{2}+2 x q_{2}(x) t^{3}+\cdots+2 x q_{n-1}(x) t^{n}+\cdots,
\end{gathered}
$$

and

$$
t^{2} f(t, x)=q_{0}(x) t^{2}+q_{1}(x) t^{3}+q_{2}(x) t^{4}+\cdots+q_{n-2}(x) t^{n}+\cdots
$$

So, we get

$$
f(t, x)\left(1-2 x t-t^{2}\right)=q_{0}(x)+\left[q_{1}(x)-2 x q_{0}(x)\right] t
$$

Thus, we obtain

$$
f(t, x)=\frac{1-x t}{1-2 x t-t^{2}}
$$

This completes the proof.

We now give the proof of the Binet formula for the modified Pell polynomials in the following theorem.

Theorem 2. The nth term of the modified Pell polynomials is

$$
q_{n}(x)=x \frac{\alpha^{n}(x)+\beta^{n}(x)}{\alpha(x)+\beta(x)}
$$

where $\alpha(x)=x+\sqrt{x^{2}+1}$ and $\beta(x)=x-\sqrt{x^{2}+1}$ are the roots of the equations $r^{2}-$ $2 x r-1=0$.

Proof of Theorem 2. We know that the general solution for the recurrence relation is given by

$$
q_{n}(x)=c \alpha^{n}(x)+d \beta^{n}(x)
$$

for some coefficients $c$ and $d$. 
Using the initial values $q_{0}(x)=c+d$ and $q_{1}(x)=c \alpha(x)+d \beta(x)$, we have

$$
c=\frac{1}{2} \text { and } d=\frac{1}{2} \text {. }
$$

Hence, the Binet formula for $q_{n}(x)$ is obtained as

$$
q_{n}(x)=x \frac{\alpha^{n}(x)+\beta^{n}(x)}{\alpha(x)+\beta(x)}
$$

So, the proof is completed.

We now investigate some identities for the modified Pell polynomials.

Theorem 3. Let $n$ and $r$ be two positive integers. Then Catalan identity for the modified Pell polynomials is

$$
q_{n+r}(x) q_{n-r}(x)-q_{n}^{2}(x)=(-1)^{n+1}+(-1)^{n-r} \frac{\left[\alpha^{r}(x)+\beta^{r}(x)\right]^{2}}{4} .
$$

Proof. By using the Binet formula of the modified Pell polynomials, we get

$$
\begin{aligned}
& q_{n+r}(x) q_{n-r}(x)-q_{n}^{2}(x) \\
& =x^{2}\left[\frac{\alpha^{n+r}(x)+\beta^{n+r}(x)}{\alpha(x)+\beta(x)}\right]\left[\frac{\alpha^{n-r}(x)+\beta^{n-r}(x)}{\alpha(x)+\beta(x)}\right]-x^{2}\left[\frac{\alpha^{n}(x)+\beta^{n}(x)}{\alpha(x)+\beta(x)}\right]^{2} \\
& =\frac{2 x^{2}(-1)^{n+1}+x^{2}(-1)^{n-r}\left[\alpha^{2 r}(x)+\beta^{2 r}(x)\right]}{[\alpha(x)+\beta(x)]^{2}}
\end{aligned}
$$

Since $\alpha+\beta=2 x$, we obtain

$$
\begin{aligned}
q_{n+r}(x) q_{n-r}(x)-q_{n}^{2}(x) & =\frac{4 x^{2}(-1)^{n+1}}{4 x^{2}}+x^{2}(-1)^{n-r} \frac{\left[\alpha^{r}(x)+\beta^{r}(x)\right]^{2}}{4 x^{2}} \\
& =(-1)^{n+1}+(-1)^{n-r} \frac{\left[\alpha^{r}(x)+\beta^{r}(x)\right]^{2}}{4}
\end{aligned}
$$

By taking $r=1$ in Theorem 3., Cassini identity for the modified Pell polynomials, which is given in the following corollary, is obtained.

Corollary 1. For positive integer $n$, we have

$$
q_{n+1}(x) q_{n-1}(x)-q_{n}^{2}(x)=(-1)^{n+1}\left(x^{2}+1\right) .
$$

D’Ocagne identity for the modified Pell polynomials is given in the following theorem.

Theorem 4. For positive integers $m$ and $n$, we get

$$
q_{m}(x) q_{n+1}(x)-q_{n}(x) q_{m+1}(x)=\left(x^{2}+1\right)(-1)^{n+1} P_{m-n}(x)
$$


where $P_{n}(x)$ is the nth Pell polynomial.

Proof. By using the Binet formula, we get

$$
\begin{aligned}
& q_{m}(x) q_{n+1}(x)-q_{n}(x) q_{m+1}(x) \\
& =x^{2}\left[\frac{\alpha^{m}(x)+\beta^{m}(x)}{\alpha(x)+\beta(x)}\right]\left[\frac{\alpha^{n+1}(x)+\beta^{n+1}(x)}{\alpha(x)+\beta(x)}\right]-x^{2}\left[\frac{\alpha^{n}(x)+\beta^{n}(x)}{\alpha(x)+\beta(x)}\right]\left[\frac{\alpha^{m+1}(x)+\beta^{m+1}(x)}{\alpha(x)+\beta(x)}\right] \\
& =x^{2} \frac{\alpha^{m}(x) \beta^{n+1}(x)+\alpha^{n+1}(x) \beta^{m}(x)-\alpha^{n}(x) \beta^{m+1}(x)-\alpha^{m+1}(x) \beta^{n}(x)}{[\alpha(x)+\beta(x)]^{2}} \\
& =x^{2} \frac{[\alpha(x)-\beta(x)]\left[\alpha^{n}(x) \beta^{m}(x)-\alpha^{m}(x) \beta^{n}(x)\right]}{[\alpha(x)+\beta(x)]^{2}} \\
& =(-1)^{n+1}\left(x^{2}+1\right) .
\end{aligned}
$$

The following theorem gives Gelin-Cesaro identity for the modified Pell polynomials.

Theorem 5. The identity

$$
\begin{aligned}
q_{n}{ }^{4}(x)-q_{n-2}(x) q_{n-1}(x) q_{n+1}(x) q_{n+2}(x)= & 4 x^{2}\left(x^{4}+2 x^{2}+1\right) \\
& -\left(4 x^{4}+3 x^{2}-1\right)(-1)^{n} \frac{\left[\alpha^{n}(x)+\beta^{n}(x)\right]^{2}}{4}
\end{aligned}
$$

where $q_{n}(x)$ is a the modified Pell polynomials.

Proof. For the equality, from the Binet formula

$$
\begin{aligned}
& q_{n}{ }^{4}(x)-q_{n-2}(x) q_{n-1}(x) q_{n+1}(x) q_{n+2}(x) \\
& =x^{4}\left[\frac{\alpha^{n}(x)+\beta^{n}(x)}{\alpha(x)+\beta(x)}\right]^{4}-x^{4}\left[\frac{\alpha^{n-2}(x)+\beta^{n-2}(x)}{\alpha(x)+\beta(x)}\right]\left[\frac{\alpha^{n-1}(x)+\beta^{n-1}(x)}{\alpha(x)+\beta(x)}\right] \\
& \quad \times\left[\frac{\alpha^{n+1}(x)+\beta^{n+1}(x)}{\alpha(x)+\beta(x)}\right]\left[\frac{\alpha^{n+2}(x)+\beta^{n+2}(x)}{\alpha(x)+\beta(x)}\right] \\
& =x^{4} \frac{\left(4-16 x^{4}-12 x^{2}\right)(-1)^{n}\left[\alpha^{n}(x)+\beta^{n}(x)\right]^{2}+\left(64 x^{6}+128 x^{4}+64 x^{2}\right)}{[\alpha(x)+\beta(x)]^{4}} \\
& =\left(4 x^{6}+8 x^{4}+4 x^{2}\right)-\frac{\left(4 x^{4}+3 x^{2}-1\right)(-1)^{n} x^{2}\left[\alpha^{n}(x)+\beta^{n}(x)\right]^{2}}{4 x^{2}} \\
& =4 x^{2}\left(x^{4}+2 x^{2}+1\right)-\left(4 x^{4}+3 x^{2}-1\right)(-1)^{n} \frac{\left[\alpha^{n}(x)+\beta^{n}(x)\right]^{2}}{4}
\end{aligned}
$$

can be written which is desired. 
We now investigate some sum formulas of this sequence.

Theorem 6. The sum of the first $n$ terms of the modified Pell polynomials is

$$
\sum_{k=1}^{n} q_{k}(x)=\frac{1}{2 x}\left[q_{n+1}(x)+q_{n}(x)-x-1\right] .
$$

Proof. From the recursive relation related with the modified Pell polynomials, we can write

$$
q_{n-1}(x)=\frac{1}{2 x} q_{n}(x)-\frac{1}{2 x} q_{n-2}(x)
$$

Then we have

$$
\begin{gathered}
q_{1}(x)=\frac{1}{2 x} q_{2}(x)-\frac{1}{2 x} q_{0}(x) \\
q_{2}(x)=\frac{1}{2 x} q_{3}(x)-\frac{1}{2 x} q_{1}(x) \\
q_{3}(x)=\frac{1}{2 x} q_{4}(x)-\frac{1}{2 x} q_{2}(x) \\
\vdots \\
q_{n}(x)=\frac{1}{2 x} q_{n+1}(x)-\frac{1}{2 x} q_{n-1}(x)
\end{gathered}
$$

Hence, we obtain

$$
\begin{aligned}
\sum_{k=0}^{n} q_{k}(x) & =\frac{1}{2 x}\left[q_{n+1}(x)+q_{n}(x)\right]-\frac{1}{2 x}\left[q_{0}(x)+q_{1}(x)\right] \\
& =\frac{1}{2 x}\left[q_{n+1}(x)+q_{n}(x)-x-1\right]
\end{aligned}
$$

which completes the proof.

From Theorem 6., we can give the following corollary.

Corollary 2. For $n \geq 1$, we have
i. $\sum_{k=1}^{n} q_{2 k}(x)=\frac{1}{2 x}\left[q_{2 n+1}(x)-x\right]$,
ii. $\sum_{k=1}^{n} q_{2 k-1}(x)=\frac{1}{2 x}\left[q_{2 n}(x)-1\right]$. 


\section{CONCLUSION}

In this study, we investigate some properties of the modified Pell polynomials. We give the proof of the Binet formula and generating function of the modified Pell polynomials. We also give some summation formulas for these polynomials. Moreover, we obtain some well-known identities, such as Catalan, Cassini, d'Ocagne and Gelin-Cesaro identities involving the modified Pell polynomials.

\section{References}

[1] V.E. Hoggatt Jr., Fibonacci and Lucas Numbers (Houghton Mifflin, Boston, 1969).

[2] T. Koshy, Fibonacci and Lucas Numbers with Applications (Wiley-Interscience, New York, 2001) pp. 51-131.

[3] S. Vajda, Fibonacci \& Lucas Numbers and The Golden Section: Theory and Applications (John Wiley and Sons, New York, 1989) pp. 9-61.

[4] T. Koshy, Pell and Pell-Lucas Numbers with Applications (Springer, New York, 2014).

[5] A.F. Horadam, Applications of modified Pell numbers to representations. Ulam Quarterly, 3(1) (1994) 34-53.

[6] R. Melham, Sums involving Fibonacci and Pell numbers. Portugaliae Mathematica, 56(3) (1999) 309-317.

[7] S. Halıcı and A. Daşdemir, On some relationships among Pell, Pell-Lucas and modified Pell sequences. Sakarya University Journal of Science, 14(2) (2010) 141-145.

[8] Y. Yuan and W. Zhang, Some identities involving the Fibonacci polynomials. The Fibonacci Quarterly, 40 (2002) 314-318.

[9] B.G.S. Doman and J.K. Williams, Fibonacci and Lucas polynomials. Mathematical Proceedings of the Cambridge Philosophical Society, 90(3) (1981) 385-387.

[10] A. Lupas, A guide of Fibonacci and Lucas polynomial. Octagon Mathematics Magazine, 7(1) (1999) 2-12.

[11] W.A. Webb and E.A. Parberry, Divisibility properties of Fibonacci polynomials. The Fibonacci Quarterly, 7(5) (1969) 457-463.

[12] A.F. Horadam and Bro. J.M. Mahon, Pell and Pell-Lucas polynomials. The Fibonacci Quarterly, 23(1) (1985) 7-20.

[13] T. Horzum and E. G. Kocer, On Some Properties of Horadam Polynomials, International Mathematical Forum, 4(25) (2009) 1243-1252. 